\section{Vomiting-control medication: when, how and why}

\section{Caroline Boothroyd}

Nausea and vomiting may be caused by:

- Various conditions

- Prescribed medications (e.g. chemotherapy)

- Motion sickness.

Physiology of vomiting (Figure 1):

- Indirect/peripheral - blood borne substances and neurological input from the vestibular nucleus cause dopamine release in the chemoreceptor trigger zone (CRTZ) and indirect stimulation of the vomiting centre

- Direct/central - cerebral disease and disease or irritation of the Gl tract, abdominal organs and the peritoneum can directly stimulate the vomiting centre

- Once the vomiting centre is stimulated a set of reflex actions are coordinated to cause vomiting.

\section{FREQUENTLY USED ANTI-EMETICS}

(Information taken from Plumbs Veterinary Drugs, BSAVA Formulary and the medication data sheet.)

\section{Maropitant}

The safety not been established in dogs less than 8 weeks old or cats less than 16 weeks old or in pregnant and lactating cats and dogs.

- Acts at the vomiting centre; therefore, effective for peripheral or central mechanisms

- Licensed for subcutaneous or intravenous injection

- Intravenous administration should be given as a bolus without mixing with other fluids

- Injection may be administered for up to 5 days and tablets for up to 14 days

- Oral dose is higher because of decreased bioavailability of the oral tablet. Cerenia ${ }^{\circledR}$ can be given the night before chemotherapy administration and is indicated for vomiting due to motion sickness in dogs

- Not for use in patients suspected of toxin ingestion, as this may allow more time for toxin absorption. Additionally, use should be delayed until gastrointestinal (GI) obstruction is ruled out

- Rarely, anaphylactic-type reactions may occur

- Metabolized by the liver so use with caution in animals with liver disease.

\section{Metoclopramide}

- Stimulates upper GI motility and has anti-emetic properties; more potent as an anti-emetic than a prokinetic agent in dogs

- Anti-emetic effects of metoclopramide are secondary to both central and peripheral effects. In the central nervous system (CNS), metoclopramide apparently antagonizes dopamine $\left(D_{2}\right)$ at receptor sites. Cats reportedly have few CNS dopamine receptors; therefore, metoclopramide may be a poor anti-emetic choice

- Adverse effects: changes in mentation and behaviour, constipation

- Several potentially serious drug interactions

- Contraindicated in patients with Gl hemorrhage, Gl obstruction, or perforation (should be ruled out before use)

- It is relatively contraindicated in patients with seizure disorders or head trauma

- In patients with phaeochromocytoma, metoclopramide may induce a hypertensive crisis

- Suitable for subcutaneous, intramuscular and intravenous injection (bolus or continusous-rate infustion) (Vomend ${ }^{\circledR}$ not licenced for intravenous use)

- Injection is light sensitive.

\section{Ondansetron}

Ondansetron has action both centrally and peripherally.

- Use is indicated when vomiting/nausea not controlled by licensed products

- No licensed product

- Dose: varies for cats and dogs, and any concurrent disease.

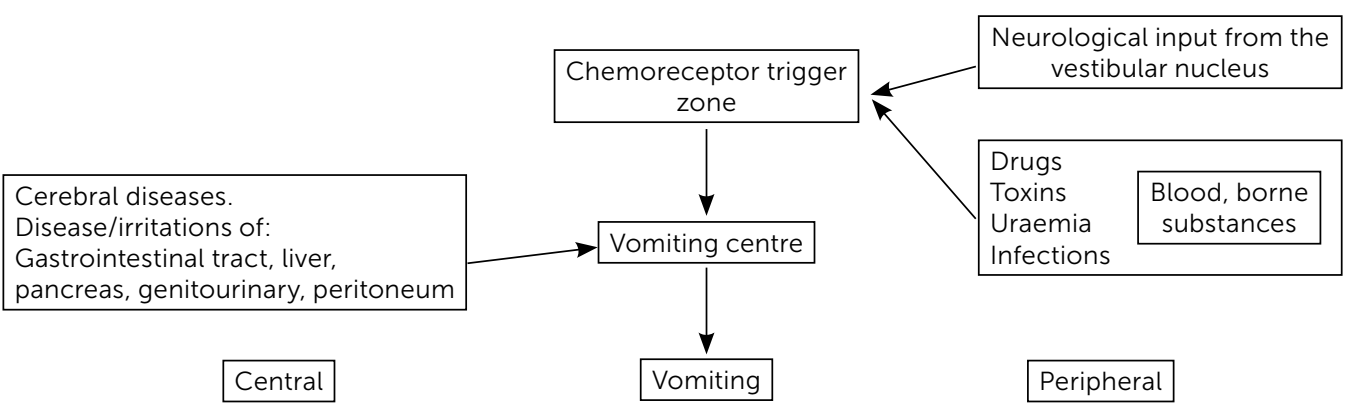

Figure 1: Physiology of vomiting.

(Figure courtesy of BSAVA Manual of Canine \& Feline Gastroenterology. Second edition) 


\section{Canine medicine}

\section{OTHER LESS COMMONLY USED MEDICATIONS}

\section{Cisapride}

- No known anti-emetic effects, but it helps to enhance gastric emptying

- Potential risk of cardiac arrhythmias in humans so gloves should be worn when administering

- No licensed product.

\section{Domperidone}

- Potent anti-emetic, similar mechanism to metoclopramide, but with fewer adverse CNS effects as it does not cross the blood-brain barrier

- No licensed product.

\section{Prochlorperazine}

- Anti-emetic activity is due to its effects primarily in the brain's emetic center and chemoreceptor trigger zone, but it also has some peripheral activity

- Used to control motion sickness and emesis associated with vestibular disease

- No licensed product

- Adverse effects: sedation or hypotension.

\section{Propantheline}

- A peripherally acting anti-emetic

- No licensed product.

\section{KEY LEARNING OBJECTIVES}

- To understand how vomiting occurs

- To understand the difference between the anti-emetics available

- To know which medications are licensed for use

\section{MULTIPLE CHOICE QUESTIONS}

1. Where is the emetic/vomiting centre located?
(A) Medulla oblongata of the brainstem
(B) The pancreas
(C) Cerebral cortex
(D) Gastrointestinal tract

2. Cerenia ${ }^{\circledR}$ is the trade name for which medication?
(A) Metocloprimide
(B) Ondansetron
(C) Maropitant
(D) Medetomidine

3. Which of the following is a central cause of vomiting?
(A) Toxins
(B) Medications
(C) Infections
(D) Pancreatitis

\section{The top to bottom of endoscopy: a practical approach}

\section{Laura George}

Due to the range of endoscopes becoming more readily available, the veterinary nurse should be competent in setting up, using and maintaining the different types. Both rigid and flexible endoscopes are available with the rigid option being cheaper and less fragile but of limited use.

\section{COMPONENTS}

All flexible endoscopes are constructed in a similar manner consisting of a hand piece with suction/insufflation buttons and directional dials, an insertion tube, an instrument channel and an umbilical cord that transmits light, air for insufflation and also allows for suction. The suction unit may be wall mounted or a portable unit and is connected directly to the endoscope.

A light source is required and this may be contained in a unit with an air pump or may be a separate unit requiring a cable to transmit light through the endoscope.

\section{CARE}

All endoscopes are transported in a case protecting them from damage. When not being transported flexible endoscopes are hung in a designated cupboard with the insertion tube hanging straight down. If stored in a case, the insertion tube may permanently curl and become unusable.

Endoscopes must be watertight, to prevent damage from fluid leaking into inner workings. Leak testing is performed before and after every use. A leakage tester is attached and pressurized to an appropriate level (manufacturer guidelines for specific endoscopes are available): if the level remains stable there is no leak. It is cheaper to repair a leaking endoscope if detected early rather than long-term damage from on-going undetected leaks.

Cleaning occurs immediately after use to avoid mucus drying in the instrument channel, which can cause corrosion. Prior to cleaning it is important to establish if the endoscope can be submerged in cleaner, a blue ring around the hand piece generally indicates it is safe but due to the risk of damage it is prudent to check with the manufacturer. An appropriate-sized cleaning brush is passed down the instrument channel ensuring it is thoroughly cleaned. Endoscopes are then disinfected between patients reducing cross-contamination.

There are various sterilization methods for endoscopic equipment including ethylene oxide gas, it is important to consult the manufacturer checking which methods are suitable. Flexible endoscopes are rarely compatible with steam sterilization due to the high temperatures required which cause extensive damage. 